\title{
Associação da Intensidade de Dor no Tempo Até à Morte dos Doentes Oncológicos Referenciados aos Cuidados Paliativos
}

\author{
Pain Intensity and Time to Death of Cancer Patients \\ Referred to Palliative Care
}

\author{
Pedro BARATA® ${ }^{1}$, Filipa SANTOS ${ }^{2}$, Graça MESQUITA³ , Alice CARDOSO ${ }^{4}$, Maria Paula CUSTÓDIO ${ }^{1}$, Marta ALVES $^{5}$, \\ Ana Luísa PAPOILA ${ }^{5}$, António BARBOSA ${ }^{6}$, Peter LAWLOR ${ }^{7}$ \\ Acta Med Port 2016 Nov;29(11):694-701 - http://dx.doi.org/10.20344/amp.7557
}

\section{RESUMO}

Introdução: A dor é uma experiência frequente nos doentes com cancro, especialmente naqueles em fase final de vida. Com este estudo, pretendemos estudar a intensidade de dor nos doentes com cancro avançado, referenciados aos cuidados paliativos, analisar os factores associados à ocorrência de dor moderada ou intensa e avaliar a sua relação com o tempo até à morte destes doentes.

Material e Métodos: Estudo prospectivo observacional que incluiu consecutivamente todos os doentes referenciados aos cuidados paliativos com tumores sólidos metastizados e sem tratamento oncológico específico. Foi considerada a intensidade de dor da escala de Edmonton, de acordo com a graduação zero a 10, onde 0 = ausência de dor e 10 = máxima dor possível.

Resultados: Entre outubro de 2012 e junho de 2015, foram incluídos 301 doentes, com idade mediana de 69 anos (37 - 94), 57\% homens e $64,8 \%$ dos doentes com performance status $3 / 4$. Aproximadamente $42 \%$ dos doentes apresentaram dor $\geq 4$ e cerca de $74,4 \%$ estavam medicados com analgesia opióide. A intensidade de dor esteve associada ao performance status dos doentes, de acordo com a análise multivariável (OR: 1,7; IC 95\%: 1,0 - 2,7; $p=0,045$ ). A mediana do tempo de sobrevivência foi de 37 dias (IC $95 \%: 28$ - 46), tendo os doentes com dor moderada ou intensa (intensidade de dor $\geq 4$ ) uma mediana de sobrevivência de 29 dias (IC $95 \%$ : 21 - 37), comparada com os 49 dias (IC 95\%: 35 - 63) para os doentes sem dor ou dor ligeira $(p=0,022)$.

Discussão: O performance status, para além de ter estado associado a uma maior intensidade de dor, esteve associado a um menor tempo até à morte dos doentes com cancro avançado referenciados aos cuidados paliativos. Também o internamento, a presença de metastização intra-abdominal e a analgesia opióide estiveram associados de forma negativa ao tempo até à morte destes doentes. Conclusão: A dor oncológica continua a ser um problema clinicamente relevante nos doentes com cancro avançado.

Palavras-chave: Cuidados Paliativos; Medição da Dor; Neoplasias.

\section{ABSTRACT}

Introduction: Pain is a common symptom experienced by cancer patients, especially in those with advanced disease. Our aim was to describe pain intensity in advanced cancer patients, referred to the palliative care unit, the factors underlying moderate to severe pain and its prognostic values.

Material and Methods: This was a prospective observational study. All patients with mestastatic solid tumors and with no specific oncologic treatment were included. Pain intensity was accessed using the pain scale from Edmonton Symptom Assessment Scale, rated from 0 to 10 on a numerical scale, where zero $=$ no pain and $10=$ worst possible pain .

Results: Between October 2012 and June 2015, a total of 301 patients participated in the study. The median age was 69 years, (3794 ); most of the patients were men (57\%) and $64.8 \%$ had a performance status of $3 / 4$. About $42 \%$ reported pain severity $\geq 4$ and $74 \%$ were medicated with opioids. Multivariate analysis indicated a correlation between performance status and reported pain (OR: 1.7; IC 95\%: $1.0-2.7 ; p=0.045$ ). Median overall survival was 37 days (IC 95\%: 28 - 46). Patients reporting moderate to severe pain (pain severity $\geq 4$ ) had a median survival of 29 days (IC 95\%: 21 - 37), comparing with those who had no or moderate pain with median survival of 49 days (IC 95\%: 35 - 63) ( $p=0.022)$.

Discussion: The performance status was associated with more intense pain. The performance status, hospitalization, intra-abdominal metastization and opioid analgesia were associated with shorter time to death in advanced cancer patients referred to palliative care. Conclusion: Cancer pain continues to be a major clinical problem in advanced cancer patients.

Keywords: Neoplasms; Pain Measurement; Palliative Care.

\section{INTRODUÇÃO}

A dor é uma experiência subjetiva e multidimensional, frequentemente relatada nos doentes oncológicos, principalmente nos que se encontram numa fase mais avançada da sua doença..$^{1-3}$ Do mau controlo sintomático que está asfrequentemente surge descrito como o mais angustiante, tendo elevado impacto negativo na qualidade de vida e deteriorando a capacidade do doente em aderir ao tratamento oncológico ou alcançar uma morte tranquila. ${ }^{1,4-8}$ sociado à fase avançada do cancro, a dor é o sintoma que

\footnotetext{
1. Serviço de Oncologia Médica. Centro Hospitalar Lisboa Central. Lisboa. Portugal.

2. Departamento de Ciências Biomédicas e Medicina. Universidade do Algarve. Faro. Portugal.

3. Unidade de Dor Crónica. Centro Hospitalar Lisboa Central. Lisboa. Portugal.

4. Cuidados Paliativos. Centro Hospitalar Lisboa Central. Lisboa. Portugal.

5. Gabinete de Análise Epidemiológica e Estatística. Centro de Investigação. Centro Hospitalar Lisboa Central. Lisboa. Portugal.

6. Centro de Bioética. Faculdade de Medicina. Universidade de Lisboa. Lisboa. Portugal.

7. Cuidados Paliativos. Institutos de Investigação de Bruyere e Ottawa. Universidade de Ottawa. Ottawa. Canadá.

$\bowtie$ Autor correspondente: Pedro Barata. pedrobaratamd@gmail.com

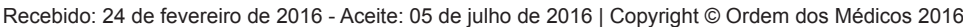


A intensidade da dor é considerada uma das dimensões clinicamente mais relevantes na experiência da dor. ${ }^{9}$ Em 1986, a Organização Mundial de Saúde (OMS) desenvolveu um protocolo para o tratamento da dor oncológica, através da classificação do tratamento por degraus - escada analgésica. ${ }^{3}$ Posteriormente, em 1999, a Veterans Health Administration propôs a avaliação da intensidade de dor enquanto 'quinto sinal vital', através de uma escala de zero a 10 , com o intuito de otimizar o controlo analgésico. ${ }^{10}$ Em Portugal, também foram criadas normas pela Direção Geral de Saúde (DGS) - Circular Normativa n. ${ }^{\circ}$ 9/DGCG de 14/06/2003 - para a avaliação e registo sistemático da intensidade de dor, pelos profissionais de saúde.

No entanto, continuam a escassear estudos prospetivos, sobretudo no contexto nacional, sobre a incidência da intensidade de dor oncológica em doença avançada. Neste sentido, propusemos estudar a intensidade de dor oncológica dos doentes referenciados aos cuidados paliativos (CP), a associação das características dos doentes e de doença com a ocorrência de dor moderada ou intensa, e avaliar a relação da intensidade de dor com o tempo até à morte destes doentes.

\section{MATERIAL E MÉTODOS}

\section{Características do hospital onde decorreu o estudo}

O Centro Hospitalar de Lisboa Central (CHLC) inclui seis hospitais localizados em Lisboa. O serviço de Oncologia Médica está fisicamente localizado no Hospital Santo António dos Capuchos (HSAC). Quando necessário, os doentes oncológicos são internados em diferentes unidades do CHLC, mais frequentemente no serviço de Medicina Interna ou Cirurgia Geral.

Os doentes oncológicos são maioritariamente referenciados ao Departamento de Cuidados Paliativos para controlo sintomático e/ou orientação do plano de cuidados futuros (muitas vezes para referenciação a unidades da rede de cuidados paliativos e cuidados domiciliários). Assim, são referenciados os doentes em ambulatório, a partir da Clínica de Oncologia do próprio centro ou doutras Instituições hospitalares, bem como os doentes internados em qualquer enfermaria do HSAC cumprindo os critérios de seleção.

\section{Amostra em estudo}

Foram incluídos consecutivamente, de outubro de 2012 a junho de 2015, todos os doentes oncológicos com tumores sólidos metastizados, sem tratamento oncológico ou com suspensão permanente do mesmo, consecutivamente referenciados à equipa de $\mathrm{CP}$ do $\mathrm{CHLC}$. Foram também incluídos os doentes sujeitos a radioterapia para efeitos de paliação/controlo sintomático (por exemplo, dor óssea ou hemorragia).

Foram excluídos do estudo os doentes com tumores do foro hematológico; ou que tenham retomado algum tipo de tratamento oncológico, como quimioterapia, radioterapia ou novas terapêuticas-alvo (por exemplo, inibidor da tirosina quinase ou anticorpo monoclonal). Os doentes afásicos ou com outro tipo de incapacidade para comunicar verbalmente em português foram também excluídos.

Este estudo foi aprovado pela Comissão de Ética do CHLC e foi obtido o consentimento informado de todos os participantes.

\section{Instrumentos e métodos de recolha de dados}

A recolha de dados dos doentes foi efectuada de acordo com a seguinte sequência: inicialmente foi utilizado o the Short Portable Mental Status Questionnaire (SPMSQ) ${ }^{11}$ na sua versão portuguesa, para excluir os doentes com algum défice cognitivo (três ou mais erros para pessoas que sabem ler e escrever e quatro ou mais erros para baixo nível educacional ou analfabetos), e também aplicada a escala Confusion Assessement Method (CAM), ${ }^{12}$ na sua versão traduzida e validada para a língua portuguesa, para excluir síndrome confusional agudo (presença de uma destas condições, de acordo com as regras do instrumento: mudança súbita do estado mental e distúrbio de atenção; pensamento desorganizado; presença de alteração súbita de consciência). Seguidamente, foram recolhidos os dados referentes às características demográficas do doente, da doença oncológica e terapêutica nas últimas 24 horas, através da revisão dos registos clínicos dos participantes. Com base no exame físico, a equipa de CP efetuou ainda a avaliação do estado funcional e clínico do doente pelo Eastern Cooperative Oncology Group performance status (PS). ${ }^{13}$

Finalmente, registámos a informação referente aos sintomas dos doentes através da aplicação da escala de Edmonton (EE), na versão revista e validada para a língua portuguesa. ${ }^{14,15}$ Esta escala é habitualmente utilizada pelas equipas de CP para avaliar a gravidade de nove sintomas: dor, dispneia, falta de apetite, náuseas, fadiga, sonolência, ansiedade, depressão e bem-estar geral. Estes são graduados de zero (ausência de sintomatologia) a 10 (sintomatologia máxima). Para o presente estudo, apenas foi considerada a informação referente à dor (EEd).

No que diz respeito à terapêutica, registámos a medicação prescrita nas 24 horas anteriores à aplicação dos instrumentos, pelas seguintes classes farmacológicas: anti-eméticos, laxantes, neurolépticos, corticóides, ansiolíticos, analgésicos não opióides e opióides. A dose total de opióides nas 24 horas que antecederam a entrada no estudo, foi registada em dose equivalente de morfina oral (DEMD), em miligramas (mg), de acordo com a tabela de conversão de opióides de Sittl $\mathrm{R}$ et al.. ${ }^{16,17}$ Por outro lado, fármacos como o paracetemol, metamizol, e os anti-inflamatórios não esteróides (AINES) foram classificados como analgésicos não-opióides, de acordo com a escada analgésica da OMS para doentes oncológicos. ${ }^{18}$ Os fármacos: haloperidol, olanzapina, quetiapina, risperidona, foram classificados como neurolépticos. A medicação ansiolítica incluiu qualquer fármaco do grupo das benzodiazepinas. Não foram incluídos os indutores de sono neste grupo terapêutico. A medicação laxante foi administrada por via oral e rectal e incluiu: bisacodilo, lactulose, sene, citrato de sódio. Não foram considerados produtos não prescritos pelos 
clínicos, tais como produtos naturais ou outros.

Todos os instrumentos foram aplicados na presença do doente e preenchidos em conjunto com um entrevistador com formação em CP.

\section{Análise estatística}

As variáveis contínuas foram descritas através da mediana (min. - max.) e as variáveis categóricas foram apresentadas através de frequências e percentagens. A intensidade de dor foi categorizada nos seguintes grupos: sem dor $(E E d=0)$, dor ligeira (EEd de 1 a 3), dor moderada (EEd de 4 a 6) e dor intensa (EEd de 7 a 10). ${ }^{19,20}$ Para determinar os fatores associados à intensidade de dor através de modelos de regressão logística univariável $(p<0,25)$ e multivariável, foi efetuada uma recodificação em apenas duas categorias desta variável: intensidade de dor $<4$ e intensidade de dor $\geq 4$.

Para o estudo do tempo até à morte, foi considerado o tempo decorrido entre a data da aplicação da EEd e a data de morte ou a data da última avaliação de seguimento. $\mathrm{Na}$ análise de sobrevivência foi utilizado o estimador de Kaplan-Meier para estimar as funções de sobrevivência dos vários grupos e o teste log-rank para as comparar. Foram ainda aplicados os modelos de regressão de Cox univariável e multivariável para identificar variáveis associadas ao tempo até à morte.

Foi considerado um nível de significância $\alpha=0,05$. A análise estatística foi realizada utilizando o software Statistical Package for Social Sciences (SPSS Inc., Chicago, IL) versão 21.0 .

\section{RESULTADOS}

O questionário de avaliação sintomática foi aplicado a 318 doentes com cancro metastático, consecutivamente referenciados aos CP. Na análise final foram incluídos 301 doentes (Fig. 1).

A idade mediana foi de 69 anos (mínimo 37, máximo 94) e 172 doentes $(57,1 \%)$ eram do sexo masculino. Dois terços dos doentes $(66,1 \%)$ tinham um nível de escolaridade básico ou inferior e $64,8 \%$ dos doentes tinham um performance status de 3 ou 4. As localizações dos tumores primários mais frequentes foram o sistema gastro-intestinal $(27,6 \%)$, pulmão $(17,6 \%)$, mama $(15,6 \%)$ e sistema génito-urinário $(13,0 \%)$. A maioria dos doentes tinha dois ou mais locais de metastização (Tabela 1). A cavidade intra-abdominal e o osso foram os locais mais frequentes de metastização, em $61 \%$ e $59 \%$ dos casos, respetivamente. A maioria dos doentes $(62 \%)$ foi previamente tratada com quimioterapia, radioterapia ou recebeu tratamento alvo para a sua doença oncológica.

A informação sobre a intensidade de dor e analgesia opióide, bem como da medicação concomitante, encontra-se descrita na Tabela 2. No grupo de doentes com dor $\geq$ $4(41,5 \%)$, aproximadamente dois terços $(74,4 \%)$ estavam medicados com analgésicos opióides (DDEM = $60 \mathrm{mg}$ ), mas apenas $20,8 \%$ foram acompanhados em consultas de Dor.

O resultado da análise univariável entre a ocorrência de dor $\geq 4$ e as características demográficas e da doença oncológica é apresentado na Tabela 3. Para a análise multivariável foram selecionadas as variáveis género, performance status, metástases ósseas e metástases do SNC (valor $p<0,25)$. No modelo final apenas permaneceu a variável

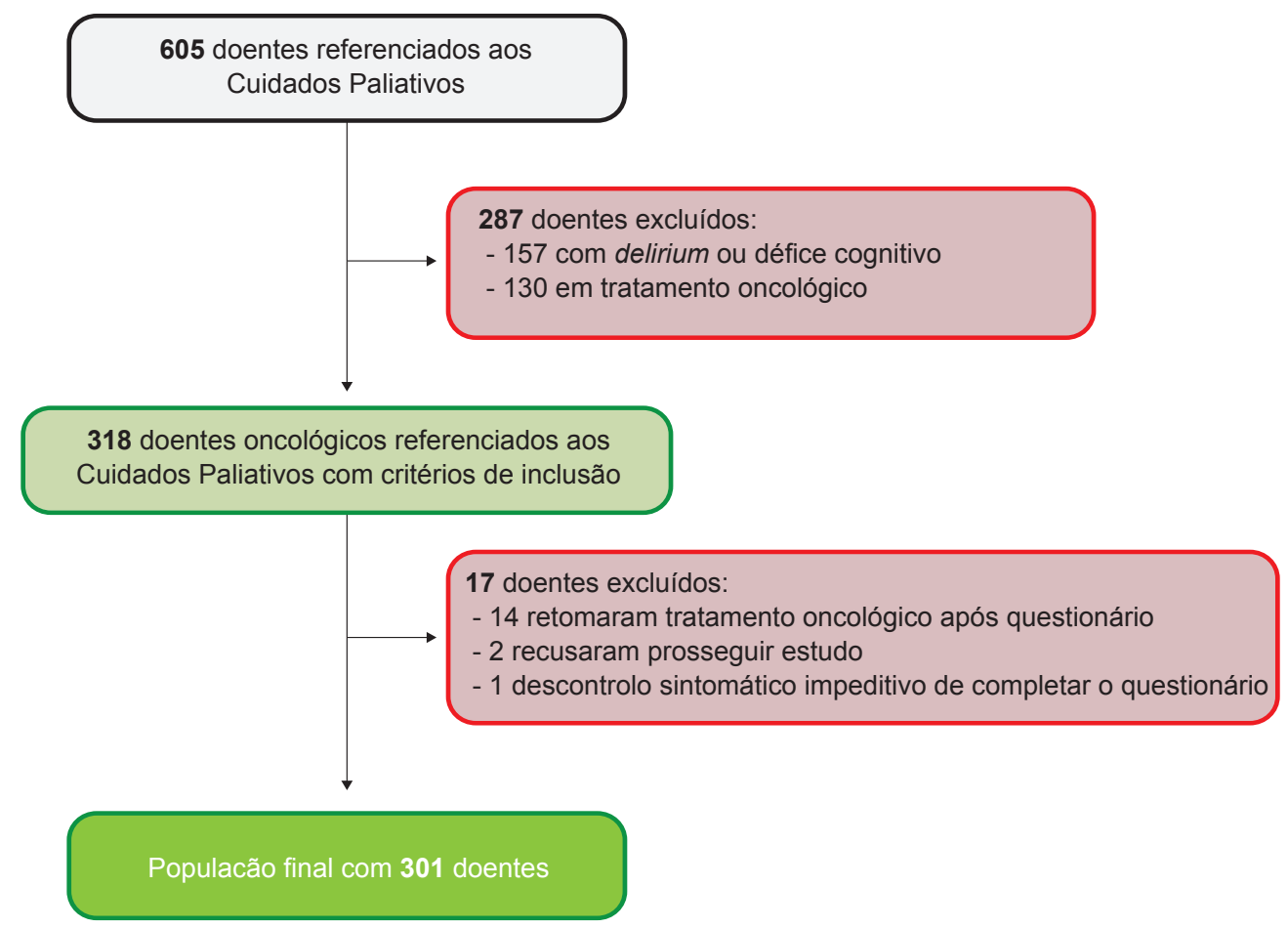

Figura 1 - Fluxo de constituição da coorte em estudo 
Tabela 1 - Características dos doentes em estudo

\begin{tabular}{|c|c|}
\hline Características clínicas & Número $(\%)$ de do€ \\
\hline \multicolumn{2}{|l|}{ Género } \\
\hline Masculino & $172(57,1)$ \\
\hline Feminino & $129(42,9)$ \\
\hline \multicolumn{2}{|l|}{ Idade } \\
\hline Mediana (anos) & 69 \\
\hline Limites & $37-94$ \\
\hline \multicolumn{2}{|l|}{ Escolaridade } \\
\hline Sem escolaridade & $30(10,0)$ \\
\hline Ensino básico & $169(56,1)$ \\
\hline Ensino secundário & $67(22,2)$ \\
\hline Ensino médio & $12(4,0)$ \\
\hline Ensino superior & $16(5,3)$ \\
\hline Desconhecido & $7(2,3)$ \\
\hline \multicolumn{2}{|l|}{ PS } \\
\hline 0 & $3(1,0)$ \\
\hline 1 & $15(5,0)$ \\
\hline 2 & $88(29,2)$ \\
\hline 3 & $142(47,2)$ \\
\hline 4 & $53(17,6)$ \\
\hline \multicolumn{2}{|l|}{ Hospitalização } \\
\hline Ambulatório & $106(35,2)$ \\
\hline Internado & $195(64,8)$ \\
\hline \multicolumn{2}{|l|}{ Local tumor primitivo } \\
\hline Vias biiares & $12(4,0)$ \\
\hline Mama & $47(15,6)$ \\
\hline Gastrointestinal & $83(27,6)$ \\
\hline Genitourinário & $39(13,0)$ \\
\hline Ginecológico & $13(4,3)$ \\
\hline Hepatocarcinoma & $14(4,7)$ \\
\hline Pulmão & $53(17,6)$ \\
\hline Pâncreas & $21(7,0)$ \\
\hline Desconhecido & $9(3,0)$ \\
\hline Outro* & $10(3,3)$ \\
\hline
\end{tabular}

Número de locais de metástases

$\begin{array}{cc}0 & 13(4,3) \\ 1 & 37(12,3) \\ >1 & 251(83,3)\end{array}$

Tratamento oncológico prévio**

\section{Realizado}

Não realizado

Tempo fim tratamento oncológico até inclusão no estudo

$$
\text { Mediana, dias (limites) }
$$$$
37(0-684)
$$

\footnotetext{
* Este grupo inclui os tumores do sistema nervoso central, cabeça e pescoço, pele, pleura e sarcomas.

** O tratamento oncológico inclui quimioterapia, radioterapia ou terapêutica-alvo dirigida ao tumor.
}

Tabela 2 - Variável dor e medicação concomitante da coorte em estudo

\begin{tabular}{lc}
\hline Intensidade de dor & Número $(\%)$ de doentes \\
Mediana (limites) & $3(0-10)$ \\
Sem dor & $106(35,2)$ \\
Dor ligeira (<4) & $70(23,3)$ \\
Dor moderada (4 - 6) & $63(20,9)$ \\
Dor intensa (7 - 8) & $23(7,6)$ \\
Medicação & \\
Analgesia opióide & $168(55,8)$ \\
DDEM (mg), mediana (limites) & $60(10-1210)$ \\
Analgesia não-opióide & $167(55,5)$ \\
Anti-eméticos & $129(42,9)$ \\
Laxantes & $118(39,2)$ \\
Corticoterapia & $100(33,2)$ \\
Ansiolíticos & $121(40,2)$ \\
Neurolépticos & $59(19,6)$ \\
Doentes seguidos em consulta de Dor & $44(14,6)$
\end{tabular}

performance status (OR: 1,7; IC 95\%: 1,0 - 2,7; $p=0,045$ ).

$\mathrm{Na}$ Tabela 4 podemos verificar o resultado da análise univariável entre as características demográficas e da doença oncológica e o tempo até à morte dos doentes. Pela análise multivariável, observou-se que a idade $\geq 70$ anos (HR: 1,3; IC 95\%: 1,1 - 1,7; $p=0,018$ ), o performance status > 2 (HR: 1,7; IC 95\%: 1,3 - 2,2; $p<0,001$ ), a presença de metastização intra-abdominal (HR: 1,4; IC 95\%: 1,1 - 1,8; $p=0,016$ ), a hospitalização na admissão (HR: 1,3; IC 95\%: $1,0-1,7 ; p=0,035)$ e a ocorrência de dor moderada ou intensa (HR: 1,4; IC 95\%: $1,1-1,7 ; p=0,014$ ), estão associadas a um maior risco de ocorrência de morte. Por fim, o tratamento prévio com quimioterapia ou radioterapia, o tratamento analgésico com fármacos não-opióides, e a presença de metastização extra-abdominal não influenciaram o tempo de sobrevivência global.

A mediana do tempo de sobrevivência dos doentes foi de 37 dias (IC 95\%: 28 - 46). Os doentes com dor moderada ou intensa $(E E d \geq 4)$ tiveram uma mediana de sobrevivência de 29 dias (IC 95\%: 21 - 37), enquanto que para os doentes com dor ligeira ou sem dor foi de 49 dias (IC 95\%: 35 - 63) $(p=0,022)$ (Fig. 2). Os doentes com performance status 3 ou 4 tiveram uma mediana de sobrevivência de 25 dias (IC 95\%: 19 - 31), comparado com os 86 dias (IC 95\%: 49 - 123) daqueles com performance status $\leq 2(p<0,001)$. Já os doentes com metástases intra-abdominais tiveram uma mediana de sobrevivência de 33 dias (IC 95\%: 21 45), comparado com 39 dias (IC 95\%: 24 - 54) daqueles sem este local de metastização $(p=0,013)$.

\section{DISCUSSÃO}

A dor é um dos sintomas mais frequentes e temidos nos doentes oncológicos, que quando mal controlada, interfere significativamente nas atividades diárias e influencia negativamente a qualidade de vida dos doentes. ${ }^{21,22}$ 
Tabela 3 - Análise univariável: resultado dos modelos de regressão logística para a ocorrência de dor moderada-intensa

\begin{tabular}{lccc}
\hline Variáveis & $\mathrm{N}(\%)$ & OR (IC 95\%) & Valor $p$ \\
\hline Idade ( $\geq 70)$ & $143(47,5)$ & $1,22(0,77-1,93)$ & 0,397 \\
Género (feminino) & $129(42,9)$ & $1,35(0,85-2,15)$ & 0,200 \\
Escolaridade (pelo menos secundário) ${ }^{*}$ & $95(31,6)$ & $1,10(0,67-1,80)$ & 0,697 \\
Performance status $>2$ & $197(65,4)$ & $1,66(1,01-2,72)$ & 0,045 \\
Metástases ósseas & $80(26,7)$ & $1,48(0,89-2,48)$ & 0,134 \\
Metástases do SNC & $32(10,7)$ & $1,68(0,80-3,51)$ & 0,168 \\
Metástases intra-torácicas & $137(45,5)$ & $0,81(0,51-1,28)$ & 0,361 \\
Metástases intra-abdominais & $184(61,1)$ & $0,92(0,58-1,48)$ & 0,735 \\
Analgesia opióide & $168(55,8)$ & $3,91(2,37-6,46)$ & $<0,001$ \\
Consulta de dor & $44(14,6)$ & $2,31(1,20-4,42)$ & 0,012 \\
Tratamento oncológico prévio (realizado) & $186(61,8)$ & $1,32(0,82-2,13)$ & 0,253 \\
Hospitalização na admissão & $194(64,7)$ & $1,19(0,73-1,92)$ & 0,490 \\
\hline
\end{tabular}

IC: Intervalo de confiança; OR: Estimativa de odds ratio; SNC: Sistema nervoso central; * Grupo de referência: ensino básico ou analfabeto. Valor $p$ foi obtido através de modelos de regressão logística.

Tabela 4 - Análise univariável: resultado dos modelos de regressão de Cox para estudar o tempo até à morte

\begin{tabular}{lcc}
\hline Variáveis & HR (IC 95\%) & valor $p$ \\
\hline Idade ( $\geq 70$ anos) & $0,68(0,54-0,86)$ & 0,001 \\
Género (feminino) & $1,03(0,81-1,31)$ & 0,818 \\
Escolaridade (pelo menos secundário)* & $1,27(0,99-1,63)$ & 0,065 \\
ECOG performance status $>2$ & $1,90(1,48-2,45)$ & 0,001 \\
Metástases ósseas & $0,96(0,74-1,26)$ & 0,766 \\
Metástases do SNC & $0,98(0,67-1,43)$ & 0,883 \\
Metástase intratorácica & $0,98(0,78-1,24)$ & 0,014 \\
Metástase intraabdominal & $1,37(1,07-1,75)$ & 0,001 \\
Analgesia opióide & $1,50(1,18-1,91)$ & 0,034 \\
Consulta de dor & $1,42(1,03-1,96)$ & 0,024 \\
Intensidade de dor $\geq 4$ & $1,32(1,04-1,67)$ & 0,790 \\
Quimioterapia & $1,03(0,81-1,32)$ & $<0,001$ \\
Hospitalização na admissão & $1,61(1,25-2,06)$ & \\
\hline
\end{tabular}

ECOG: Eastern Cooperative Oncology Group; IC: Intervalo de confiança; HR: Estimativa dos hazard ratio; SNC: Sistema nervoso central; * Grupo de referência: ensino básico ou analfabeto. Valor $p$ obtido através de modelos de regressão de Cox.

A dor é multidimensional na sua natureza, variando os seus componentes físicos, psíquicos e espirituais. Apesar de constituir apenas uma componente da experiência álgica, a intensidade de dor é, sem dúvida, uma das dimensões clinicamente mais relevantes ${ }^{23}$ e associa-se com o grau de interferência funcional sentido pelo doente, qualidade de vida, e decisões terapêuticas específicas. ${ }^{24}$ Numa revisão sistemática recente, que reuniu mais de 9760 doentes, verificou-se que cerca de um terço dos doentes com cancro têm dor no momento do diagnóstico, e entre $65 \%$ e $85 \%$ dos doentes com cancro avançado irão sentir dor. ${ }^{6} \mathrm{Na}$ nossa amostra, cerca de $42 \%$ dos doentes reportaram dor $\geq$ 4 , e mais de metade dos doentes (56\%) estava medicado com analgésicos opióides. Valorizamos a menor incidência de dor reportada no CHLC, podendo ficar a dever-se a diferentes razões. Por um lado, na instituição, existe uma proximidade física e de colaboração entre os departamentos de Oncologia Médica e Unidade de Dor. Por outro lado, desde 2011, data em que foi desenvolvido um protocolo de diagnóstico e tratamento de dor oncológica, é aplicado a todos os doentes que recorrem à urgência do serviço de Oncologia com dor não controlada. Por fim, passou a ser parte integrante da formação específica em Oncologia Médica no CHLC, o estágio clínico na referida unidade de dor. Admitimos que todas estas medidas e a colaboração existente poderão ajudar a explicar a menor incidência de dor oncológica, no caso, inferior em cerca de $20 \%-25 \%$ às publicações internacionais. No entanto, estes números ilustram a dimensão do problema que a dor ainda representa.

De entre os fatores relacionados com a intensidade de dor, destacam-se o género feminino, o PS, a metastização óssea e do SNC. Destes, apenas o PS se manteve na análise multivariável. Outros grupos de investigação já reportaram a associação entre PS e outros sintomas, como dor oncológica. ${ }^{25,26}$ Aqui, verificámos que os doentes com intensidade de dor mais elevada foram aqueles a quem 


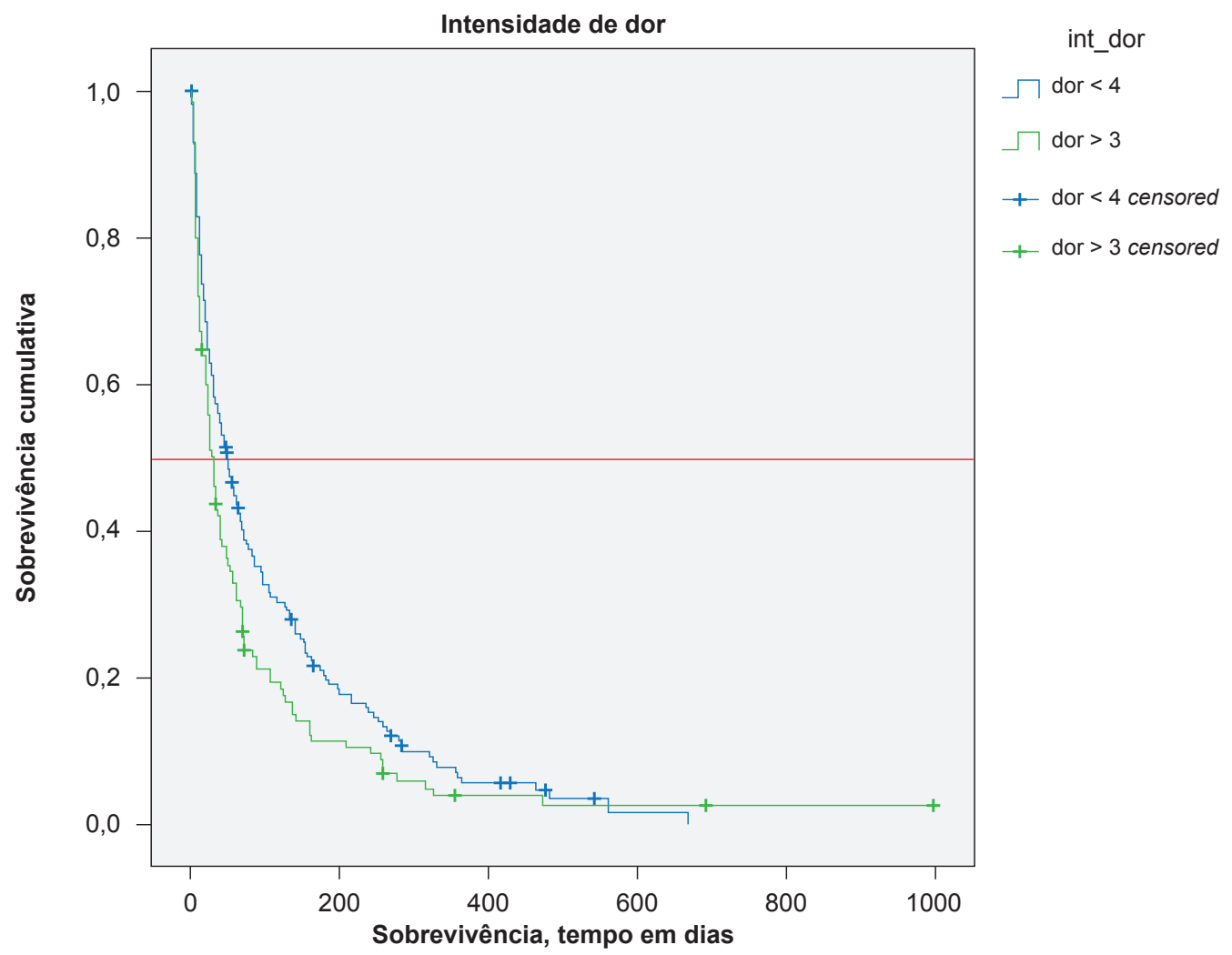

Figura 2 - Curva de sobrevivência da coorte em relação à intensidade de dor

foi prescrita mais analgesia opióide e que originaram mais pedidos de acompanhamento pela Unidade de Dor, como ficou demonstrado na análise univariável. Esta evidência clínica dispensou a incorporação destas duas variáveis no modelo multivariável.

A mediana do tempo de sobrevivência dos doentes foi inferior a 40 dias, o que é inferior ao preconizado para os doentes sem tratamento oncológico específico, que pode ultrapassar os três meses, segundo diferentes métodos de cálculo ajustado de prognóstico. ${ }^{27}$ Aproximadamente dois terços dos doentes suspendeu o tratamento oncológico nos 37 dias que antecederam a entrada no estudo, o que sugere que alguns destes doentes poderão ter recebido tratamento oncológico específico numa fase da doença em que o benefício clínico não é evidente e em que o prejuízo não é desprezável. ${ }^{28}$ Por outro lado, esta realidade contrasta com a tendência actual de integrar cada vez mais precocemente os CP no plano de tratamento da doença avançada, suportado pela evidência de benefício clinico. ${ }^{29,30}$

A intensidade de dor teve uma associação negativa ao tempo até à morte dos doentes em estudo. Esta associação foi também descrita por outros grupos de investigação, em doentes com cancro recidivado ou avançado, de localizações anatómicas específicas, como o cancro do reto ou próstata. ${ }^{31,32}$ Os doentes com dor mais intensa são também aqueles que são prescritos analgésicos de maior potência, pelo que a associação entre analgesia opióide e pior prognóstico reportada no nosso estudo, é entendida neste sentido. Por outro lado, a limitação funcional (PS) esteve também associada a um pior prognóstico dos doentes da nossa amostra. A diferença em valor absoluto é de 19 dias, o que pode ter significado clínico, tendo em conta a ordem de grandeza da sobrevivência mediana neste contexto. Esta relação directa entre o valor do performance status e o tempo de sobrevivência foi já demonstrada noutros estudos. ${ }^{27,33,34}$ Apesar de o PS ser uma escala simplista e não representativa da limitação funcional dos doentes, o resultado do nosso estudo é coerente com o esperado clinicamente, onde os doentes mais fragilizados são aqueles com menor reserva funcional e por isso, com pior prognóstico. Do mesmo modo, a necessidade de internamento dos doentes sugere uma maior deterioração clinica, seja pela doença por si ou pelo descontrolo sintomático que provoca, o que ajuda a explicar a associação do internamento dos doentes a um pior prognóstico.

Sendo um estudo de doentes com diferentes tumores sólidos e com diferentes locais de metastização, é interessante constatar que apenas a presença de metastização intra-abdominal esteve associada a pior prognóstico. Esta classificação inclui a metastização visceral ou ganglionar e pélvica, embora a relação do local de metástases (fígado, glândula supra-renal, ganglionar) com a função de órgãos fundamentais (ex: fígado) não tenha sido aqui estudada. Sabe-se que a evidência de metastização altera o prognóstico dos doentes com cancro; já o impacto da localização anatómica, sede de metástase, é muito mais difícil de ser estudado.

Importa referir alguns aspetos que limitam as conclusões a tirar deste estudo. Tendo em consideração que o foco do estudo foi a intensidade de dor, algumas das suas 
características importantes não foram recolhidas, designadamente a sua localização, tipo, ou o impacto na qualidade de vida do doente. O facto de ter sido utilizada a escala da dor do questionário de Edmonton e não uma escala multidimensional, justifica a ausência dessa informação, embora relevante.

Existem também algumas características da amostra em estudo que a podem tornar específica, e não representativa da população oncológica Portuguesa. A inclusão de doentes de uma única Instituição levou a uma diferente representação de alguns tumores primários. Admitimos que a elevada proporção de tumores do foro gastrintestinal, hepato-bilio-pancreático e pulmão não seja representativa da população oncológica; antes reflete a realidade deste Hospital, traduzindo uma realidade institucional, o que torna a sua generalização mais difícil. Seria ideal a avaliação de um grupo com um número limitado de tumores primários e/ou locais de metastização e comparar esses resultados com os obtidos no presente estudo. Por outro lado, a inclusão de doentes maioritariamente (64\%) hospitalizados, pode constituir um viés de selecção dos doentes com maior fragilidade clinica e, por isso, com performance status mais elevados. Finalmente, não explorámos o local de morte dos doentes, pese embora o seu potencial impacto no controlo sintomático e tempo até à morte nos doentes em estudo.

\section{CONCLUSÃO}

Apesar de todas as orientações de abordagem, tratamento e recomendações nacionais e internacionais existentes, a dor oncológica continua a ser um problema relevante nos doentes com cancro avançado, presente em mais de $40 \%$ dos casos.

O performance status, para além de ter estado associado a uma maior intensidade de dor, esteve associado a um menor tempo até à morte dos doentes com cancro avançado referenciados aos CP. Também o internamento, a presença de metastização intra-abdominal e a analgesia opióide estiveram associados de forma negativa ao tempo até à morte destes doentes.

O número crescente de doentes oncológicos reforça a importância em diagnosticar e reduzir a incidência de dor nestes doentes. Para o futuro, propomos estudar a eficácia da aplicação de protocolos de diagnóstico, abordagem e tratamento precoces de dor oncológica, a desenvolver pelas equipas de Unidade de Dor e CP.

\section{AGRADECIMENTOS}

Os autores gostariam de agradecer aos assistentes operacionais Flávia Pinto e Hugo Sousa o apoio administrativo prestado.

\section{PROTECÇÃO DE PESSOAS E ANIMAIS}

Este estudo foi aprovado pela Comissão de Ética do Centro Hospitalar Lisboa Central. Os procedimentos seguidos estavam de acordo com a Declaração de Helsínquia da Associação Médica Mundial.

\section{CONFIDENCIALIDADE DOS DADOS}

Os autores declaram ter seguido os protocolos do seu centro de trabalho acerca da publicação de dados.

\section{CONFLITOS DE INTERESSE}

Os autores declaram não terem qualquer conflito de interesse relativamente ao presente artigo.

\section{FONTES DE FINANCIAMENTO}

Os autores declaram não ter recebido subsídios ou bolsas para a elaboração do artigo.

\section{REFERÊNCIAS}

1. Cherny NI, Portenoy RK. The management of cancer pain. CA Cancer J Clin. 1994;44:263-303.

2. Barbosa A, Barbosa A, Neto I. Bioética e cuidados paliativos. Manual de Cuidados Paliativos: Núcleo de Cuidados Paliativos. Lisboa: Centro de Bioética da Faculdade de Medicina de Lisboa; 2006.

3. World Health Organization. Cancer pain relief: with a guide to opioid availability. Geneva: WHO; 1996.

4. Steinhauser KE, Christakis NA, Clipp EC, McNeilly M, Mclntyre L, Tulsky JA. Factors considered important at the end of life by patients, family, physicians, and other care providers. JAMA. 2000;284:2476-82.

5. Reis-Pina P, Lawlor PG, Barbosa A. Cancer-related pain management and the optimal use of opioids. Acta Med Port. 2015;28:376-81.

6. van den Beuken-van Everdingen $\mathrm{MH}$, de Rijke JM, Kessels AG, Schouten HC, van Kleef M, Patijn J. Prevalence of pain in patients with cancer: a systematic review of the past 40 years. Ann Oncol. 2007;18:1437-49.

7. Miaskowski C, Aouizerat BE, Dodd M, Cooper B. Conceptual issues in symptom clusters research and their implications for quality-oflife assessment in patients with cancer. J Natl Cancer Inst Monogr. 2007;(37):39-46.

8. Dodd MJ, Miaskowski C, Paul SM. Symptom clusters and their effect on the functional status of patients with cancer. Oncol Nurs Forum. 2001;28:465-70.

9. Frampton CL, Hughes-Webb P. The measurement of pain. Clin Oncol. 2011;23:381-6.

10. Kirsch B, Berdine H, Zablotsky D, Wenzel G, Meyer C. Management strategy: identifying pain as the fifth vital sign. Veterans Health Syst $\mathrm{J}$.

2000;5:49-59

11. Garcia CA. Doença de Alzheimer: problemas de diagnóstico clínico. Lisboa: Universidade de Lisboa; 1984.

12. Sampaio FM, Sequeira CA. Tradução e validação do Confusion Assessment Method para a população portuguesa. Rev Enferm. 2013:125-34.

13. Oken MM, Creech RH, Tormey DC, Horton J, Davis TE, McFadden ET, et al. Toxicity and response criteria of the Eastern Cooperative Oncology Group. Am J Clin Oncol. 1982;5:649-55.

14. Watanabe SM, Nekolaichuk CL, Beaumont C. The Edmonton Symptom Assessment System, a proposed tool for distress screening in cancer patients: development and refinement. Psycho-Oncol. 2012;21:977-85.

15. Monteiro DR, Almeida MA, Kruse MH. Translation and cross-cultural adaptation of the Edmonton Symptom Assessment System for use in palliative care. Rev Gaúcha Enferm. 2013;34:163-71.

16. Mercadante $S$, Caraceni A. Conversion ratios for opioid switching in the treatment of cancer pain: a systematic review. Palliat Med. 2011;25:50415.

17. Sittl R, Likar R, Nautrup BP. Equipotent doses of transdermal fentanyl and transdermal buprenorphine in patients with cancer and noncancer pain: results of a retrospective cohort study. Clin Ther. 2005;27:225-37.

18. Schug SA, Zech D, Dorr U. Cancer pain management according to WHO analgesic guidelines. J Pain Symptom Manage. 1990;5:27-32.

19. Swarm R, Abernethy AP, Anghelescu DL, Benedetti C, Blinderman CD, Boston B, et al. Adult cancer pain. J Natl Compr Cancer Netw. 2010;8:1046-86.

20. Ripamonti Cl, Santini D, Maranzano E, Berti M, Roila F. Management 
of cancer pain: ESMO Clinical Practice Guidelines. Ann Oncol. 2012;23:vii139-54.

21. Pina $P$, Sabri E, Lawlor PG. Characteristics and associations of pain intensity in patients referred to a specialist cancer pain clinic. Pain Res Manag. 2015;20:249-54

22. Te Boveldt N, Vernooij-Dassen M, Burger N, ljsseldijk M, Vissers $\mathrm{K}$, Engels $\mathrm{Y}$. Pain and its interference with daily activities in medical oncology outpatients. Pain Physician. 2013;16:379-89.

23. Clark WC, Ferrer-Brechner T, Janal MN, Carroll JD, Yang JC. The dimensions of pain: a multidimensional scaling comparison of cancer patients and healthy volunteers. Pain. 1989;37:23-32.

24. Chow E, Doyle M, Li K, Bradley N, Harris K, Hruby G, et al. Mild moderate, or severe pain categorized by patients with cancer with bone metastases. J Palliat Med. 2006;9:850-4.

25. Bradley N, Davis L, Chow E. Symptom distress in patients attending an outpatient palliative radiotherapy clinic. J Pain Symptom Manage. 2005;30:123-31.

26. Lin CC, Lai YL, Ward SE. Effect of cancer pain on performance status, mood states, and level of hope among Taiwanese cancer patients. J Pain Symptom Manage. 2003;25:29-37.

27. den Daas N. Estimating length of survival in end-stage cancer: a review of the literature. Journal Pain Symptom Manage. 1995;10:548-55.
28. Earle CC, Neville BA, Landrum MB, Ayanian JZ, Block SD, Weeks JC Trends in the aggressiveness of cancer care near the end of life. J Clin Oncol. 2004;22:315-21.

29. Temel JS, Greer JA, Muzikansky A, Gallagher ER, Admane S, Jackson VA, et al. Early palliative care for patients with metastatic non-small-cell lung cancer. N Engl J Med. 2010;363:733-42.

30. Smith TJ, Temin S, Alesi ER, Abernethy AP, Balboni TA, Basch EM, et al. American Society of Clinical Oncology provisional clinical opinion: the integration of palliative care into standard oncology care. J Clin Oncol. 2012;30:880-7.

31. You YN, Habiba H, Chang GJ, Rodriguez-bigas MA, Skibber JM. Prognostic value of quality of life and pain in patients with locally recurrent rectal cancer. Ann Surg Oncol. 2011;18:989-96.

32. Halabi S, Vogelzang NJ, Kornblith AB, Ou SS, Kantoff PW, Dawson NA et al. Pain predicts overall survival in men with metastatic castrationrefractory prostate cancer. J Clin Oncol. 2008;26:2544-9.

33. Mercadante $S$, Casuccio A, Fulfaro F. The course of symptom frequency and intensity in advanced cancer patients followed at home. J Pain Symptom Manage. 2000;20:104-12.

34. Vainio A, Auvinen A. Prevalence of symptoms among patients with advanced cancer: an international collaborative study. Symptom Prevalence Group. J Pain Symptom Manage. 1996;12:3-10. 